\title{
NEUTRON NOISE-BASED ANOMALY CLASSIFICATION AND LOCALIZATION USING MACHINE LEARNING
}

\author{
Demazière $\mathbf{C}^{1}$, Mylonakis $\mathrm{A}^{1}$, Vinai $\mathrm{P}^{1}$, Durrant $\mathrm{A}^{2}$, De Sousa Ribeiro $\mathrm{F}^{2}$, Wingate $\mathrm{J}^{2}$, \\ Leontidis $G^{2}$ and Kollias $S^{2}$ \\ ${ }^{1}$ Chalmers University of Technology \\ Department of Physics, Division of Subatomic and Plasma Physics \\ SE-412 96 Gothenburg, Sweden \\ ${ }^{2}$ University of Lincoln \\ School of Computer Science, MLearn Group \\ Lincoln, United Kingdom \\ demaz@chalmers.se, antonios.mylonakis@chalmers.se, vinai@chalmers.se, \\ ADurrant@lincoln.ac.uk, fDeSousaRibeiro@lincoln.ac.uk, JWingate@lincoln.ac.uk, \\ GLeontidis@lincoln.ac.uk, SKollias@lincoln.ac.uk
}

\begin{abstract}
A methodology is proposed in this paper allowing the classification of anomalies and subsequently their possible localization in nuclear reactor cores during operation. The method relies on the monitoring of the neutron noise recorded by in-core neutron detectors located at very few discrete locations throughout the core. In order to unfold from the detectors readings the necessary information, a 3-dimensional Convolutional Neural Network is used, with the training and validation of the network based on simulated data. In the reported work, the approach was also tested on simulated data. The simulations were carried out in the frequency domain using the CORE SIM+ diffusion-based two-group core simulator. The different scenarios correspond to the following cases: a generic "absorber of variable strength", axially travelling perturbations at the velocity of the coolant flow (due to e.g. fluctuations of the coolant temperature at the inlet of the core), fuel assembly vibrations, control rod vibrations, and core barrel vibrations. In all those cases, various frequencies were considered and, when relevant, different locations of the perturbations and different vibration modes were taken into account. The machine learning approach was able to correctly identify the different scenarios with a maximum error of $0.11 \%$. Moreover, the error in localizing anomalies had a mean squared error of 0.3072 in mesh size, corresponding to less than $4 \mathrm{~cm}$. The proposed methodology was also demonstrated to be insensitive to parasitic noise and will be tested on actual plant data in the near future.
\end{abstract}

KEYWORDS: neutron noise, machine learning, core diagnostics, core monitoring

\section{INTRODUCTION}

The monitoring of nuclear reactor cores using non-intrusive techniques is becoming increasingly important, due to the ageing fleet of reactors and, correspondingly, operational problems being more frequently encountered. In this respect, noise diagnostics, which relies on the analysis of the deviations of 
the local neutron flux from its time-averaged value, represents one of the most powerful techniques. Most of the diagnostic tasks are based on unfolding from the detector readings the nature and possibly location of the driving perturbation or anomaly. This unfolding requires the prior determination of the reactor transfer function, i.e. the function giving the response of the system to localized perturbations. If the number of detectors located throughout the core equated to the number of possible locations of the perturbations, the unfolding could be carried out by mathematically inverting the reactor transfer function. Nevertheless, the in-core instrumentation of western-type LWRs is very scarce, thus rendering the formal inversion of the reactor transfer function impossible. In addition, several types of perturbations, being either spatially localized or distributed, might coexist, further complicating the unfolding. Clearly, another solution strategy is called for.

Based upon some earlier work of the present authors [1,2], non-parametric unfolding techniques relying on machine learning are considered in this work, as part of the Horizon 2020 CORTEX project (CORe monitoring Techniques and EXperimental validation and demonstration) [3]. More specifically, a multitask, densely connected, 3D-Convolutional Neural Network (CNN), has been employed. The convolutions provide spatial feature extraction from the volumetric frequency input, classifying and localizing based on a feature vector representation of the input volume. The training, validation and testing of the CNN were all based on simulation data, with the simulations carried out with a frequency domain, diffusion-based core simulator, named CORE SIM+, developed at Chalmers University of Technology. Thanks to the non-uniform spatial mesh capabilities, CORE SIM+ has the ability to model the effect of extremely localized noise sources in large commercial cores.

The paper presents the results of CNN-based unfolding for a complete set of scenarios of possible anomalies. The paper is structured as follows. First, the simulations used for machine learning are presented. Different scenarios were considered: a generic "absorber of variable strength", axially travelling perturbations at the velocity of the coolant flow (due to e.g. fluctuations of the coolant temperature at the inlet of the core), fuel assembly vibrations, control rod vibrations, and core barrel vibrations. Thereafter, the machine learning algorithms used and their testing on the simulated data are thoroughly described. The paper ends with some conclusions and remarks on the future application of the developed methodology to actual plant data.

\section{MODELLING OF THE EFFECT OF VARIOUS ANOMALIES}

As will be explained in Section 3, machine learning is used to unfold from the neutron detector readings the possible existence and subsequent characterization of perturbations in a nuclear reactor core. Prior to the unfolding, the machine learning algorithms need to be fed with training data sets, i.e. data for which a known perturbation is assumed and the corresponding induced neutron noise at the location of the detectors is estimated.

The ability of successful unfolding thus heavily relies on covering all possible scenarios in the training data sets and on properly modelling their effect. For the latter, a frequency-domain modelling tool, CORE SIM+, developed at Chalmers University of Technology was used [4]. This tool estimates the effect of macroscopic cross-section stationary perturbations onto the neutron flux using the two-group diffusion approximation and linear theory. The spatial discretization of the balance equations is based on finite differences, assuming Cartesian geometry. Dedicated numerical methods were implemented in the tool so that extra fine mesh can be used for modelling extremely localized perturbations and their effect. With a proper set of three-dimensional nodal cross-sections and diffusion coefficients and a set of point-kinetic parameters, the tool estimates at the angular frequency $\omega$ the neutron noise $\delta \phi_{1}(\mathbf{r}, \omega)$ and $\delta \phi_{2}(\mathbf{r}, \omega)$ in the fast and thermal energy groups, respectively, solution of the following balance equations: 


$$
\begin{aligned}
& \left\{\boldsymbol{\nabla} \cdot[\mathbf{D}(\mathbf{r}) \boldsymbol{\nabla}]+\Sigma_{d y n}(\mathbf{r}, \omega)\right\} \times\left[\begin{array}{l}
\delta \phi_{1}(\mathbf{r}, \omega) \\
\delta \phi_{2}(\mathbf{r}, \omega)
\end{array}\right] \\
& =\phi_{r}(\mathbf{r}) \delta \Sigma_{r}(\mathbf{r}, \omega)+\boldsymbol{\phi}_{a}(\mathbf{r})\left[\begin{array}{l}
\delta \Sigma_{a, 1}(\mathbf{r}, \omega) \\
\delta \Sigma_{a, 2}(\mathbf{r}, \omega)
\end{array}\right]+\boldsymbol{\phi}_{f}(\mathbf{r}, \omega)\left[\begin{array}{l}
\delta v \Sigma_{f, 1}(\mathbf{r}, \omega) \\
\delta v \Sigma_{f, 2}(\mathbf{r}, \omega)
\end{array}\right]
\end{aligned}
$$

The definition of the different quantities can be found in, e.g., [4].

As can be seen from Eq. (1), the modelling of the effect of perturbations requires the prior modelling of physical perturbations in terms of macroscopic cross-section perturbations. This process is far from being trivial and is given special attention in the following. The considered scenarios are: a generic "absorber of variable strength", axially travelling perturbations at the velocity of the coolant flow (due to e.g. fluctuations of the coolant temperature at the inlet of the core), fuel assembly vibrations, control rod vibrations, and core barrel vibrations.

\subsection{Generic "absorber of variable strength"}

The first scenario being considered is a Dirac-like perturbation in point $\mathbf{r}^{\prime}$. In such a case, the right-hand side of Eq. (1) is replaced by $\delta\left(\mathbf{r}-\mathbf{r}^{\prime}\right)$ being introduced either in the fast group or in the thermal group. The corresponding solution to Eq. (1) is given by the Green's function $G_{g^{\prime} \rightarrow g}\left(\mathbf{r}, \mathbf{r}^{\prime}, \omega\right)$, thus giving the neutron noise induced in the spatial point $\mathbf{r}$ and energy group $g$ due to a Dirac-like perturbation in the spatial point $\mathbf{r}^{\prime}$ and energy group $g^{\prime}$.

It should be mentioned that the generic "absorber of variable strength" as described above might be seen as an artificial perturbation, since it is not directly related to a "physical" perturbation (as the ones described in the remaining of this Section). Nevertheless, the incentives to estimate the effect of a generic "absorber of variable strength" are twofold. First, the neutron noise induced by any type of perturbation can always be expressed as convolution integrals between the actual noise source $S_{g^{\prime}}$ with $g^{\prime}=1,2$ and the Green's function as:

$$
\left[\begin{array}{l}
\delta \phi_{1}(\mathbf{r}, \omega) \\
\delta \phi_{2}(\mathbf{r}, \omega)
\end{array}\right]=\left[\begin{array}{l}
\int\left[G_{1 \rightarrow 1}\left(\mathbf{r}, \mathbf{r}^{\prime}, \omega\right) S_{1}\left(\mathbf{r}^{\prime}, \omega\right)+G_{2 \rightarrow 1}\left(\mathbf{r}, \mathbf{r}^{\prime}, \omega\right) S_{2}\left(\mathbf{r}^{\prime}, \omega\right)\right] d^{3} \mathbf{r}^{\prime} \\
\int\left[G_{1 \rightarrow 2}\left(\mathbf{r}, \mathbf{r}^{\prime}, \omega\right) S_{1}\left(\mathbf{r}^{\prime}, \omega\right)+G_{2 \rightarrow 2}\left(\mathbf{r}, \mathbf{r}^{\prime}, \omega\right) S_{2}\left(\mathbf{r}^{\prime}, \omega\right)\right] d^{3} \mathbf{r}^{\prime}
\end{array}\right]
$$

This explains why the prior estimation of the Green's function is required. Second, in case the machine learning-based unfolding fails to identify any of the (more "physical") scenarios described in the remaining of this Section, being able to estimate the noise source in terms of a fast (i.e. $g=1$ ) or thermal (i.e. $g=2$ ) perturbation, or a combination of both, as well as its spatial spread is still of very high diagnostic value.

\subsection{Axially travelling perturbations at the velocity of the coolant flow}

In this scenario, a perturbation of the coolant is assumed. Although it is more likely that this perturbation is created outside of the core, we will consider, for the sake of generality, that the perturbation can also be created inside the core. The perturbation is assumed to travel upwards with the coolant along the $z$-axis at a velocity $v$. For simplicity, it is further assumed that the velocity is axially independent. Furthermore, the effect of the coolant perturbation is supposed to only modify the removal macroscopic cross-section. In the frequency-domain, the corresponding perturbation would have its largest effect on the removal cross-section, expressed as: 


$$
\delta \Sigma_{r}(\mathbf{r} \equiv(x, y, z), \omega)=\left\{\begin{array}{r}
0, \text { if }(x, y) \neq\left(x_{0}, y_{0}\right) \\
0, \text { if }(x, y)=\left(x_{0}, y_{0}\right) \text { and } z<z_{0} \\
\delta \Sigma_{r}\left(x_{0}, y_{0}, z_{0}, \omega\right) \exp \left[-\frac{i \omega\left(z-z_{0}\right)}{v}\right], \text { if }(x, y)=\left(x_{0}, y_{0}\right) \text { and } z \geq z_{0}
\end{array}\right.
$$

where $\left(x_{0}, y_{0}, z_{0}\right)$ represents the location in the core in which the perturbation is applied, and $z$ corresponds to the axial elevation within the core. The perturbation introduced at $\left(x_{0}, y_{0}, z_{0}\right)$ is further assumed to be white. The corresponding noise source is then given by:

$$
\left[\begin{array}{l}
S_{1}\left(\mathbf{r}^{\prime}, \omega\right) \\
S_{2}\left(\mathbf{r}^{\prime}, \omega\right)
\end{array}\right]=\phi_{r}\left(\mathbf{r}^{\prime}\right) \delta \Sigma_{r}\left(\mathbf{r}^{\prime}, \omega\right)
$$

\subsection{Fuel assembly vibrations}

In this scenario, a fuel assembly is assumed to vibrate in the $x$ - and/or $y$-direction. Concerning the vibration mode in the axial direction, several possibilities exist: the cantilevered beam mode, the simply supported on both sides mode (with its two first harmonics), and the cantilevered beam and simply supported mode (with its two first harmonics) [5]. Each vibration mode has a given axial shape and a corresponding eigenfrequency.

Because of the homogenization of the material data at the nodal level, the modelling of fuel assembly vibrations corresponds to the introduction in the $(x, y)$ plane of Dirac-like perturbations at the boundary of each homogeneous region, each perturbation being given by the difference of static macroscopic crosssections between the moving region and its neighboring region. Using the notations introduced in Fig. 1, denoting by $h$ the displacement in the $(x, y)$ plane and $\varepsilon_{x}$ and $\varepsilon_{y}$ its projection on the $x$ - and $y$ directions, respectively, the perturbation of the macroscopic cross-sections of type $\alpha$ is then given as:

$$
\begin{aligned}
& \delta \Sigma_{\alpha, g}^{x}(x, z, \omega)=h(z, \omega) \delta\left(x-a_{0}\right)\left[\Sigma_{\alpha, g, I}-\Sigma_{\alpha, g, I I}\right]+h(z, \omega) \delta\left(x-b_{0}\right)\left[\Sigma_{\alpha, g, I I}-\Sigma_{\alpha, g, I I}\right] \\
& \delta \Sigma_{\alpha, g}^{y}(y, z, \omega)=h(z, \omega) \delta\left(y-c_{0}\right)\left[\Sigma_{\alpha, g, I V}-\Sigma_{\alpha, g, I I}\right]+h(z, \omega) \delta\left(y-d_{0}\right)\left[\Sigma_{\alpha, g, I I}-\Sigma_{\alpha, g, V}\right]
\end{aligned}
$$

The noise source is then given by the following expression:

$$
\left[\begin{array}{l}
S_{1}\left(\mathbf{r}^{\prime}, \omega\right) \\
S_{2}\left(\mathbf{r}^{\prime}, \omega\right)
\end{array}\right]=\phi_{r}\left(\mathbf{r}^{\prime}\right) \delta \Sigma_{r}\left(\mathbf{r}^{\prime}, \omega\right)+\boldsymbol{\phi}_{a}\left(\mathbf{r}^{\prime}\right)\left[\begin{array}{l}
\delta \Sigma_{a, 1}\left(\mathbf{r}^{\prime}, \omega\right) \\
\delta \Sigma_{a, 2}\left(\mathbf{r}^{\prime}, \omega\right)
\end{array}\right]+\boldsymbol{\phi}_{f}\left(\mathbf{r}^{\prime}, \omega\right)\left[\begin{array}{l}
\delta v \Sigma_{f, 1}\left(\mathbf{r}^{\prime}, \omega\right) \\
\delta v \Sigma_{f, 2}\left(\mathbf{r}^{\prime}, \omega\right)
\end{array}\right]
$$




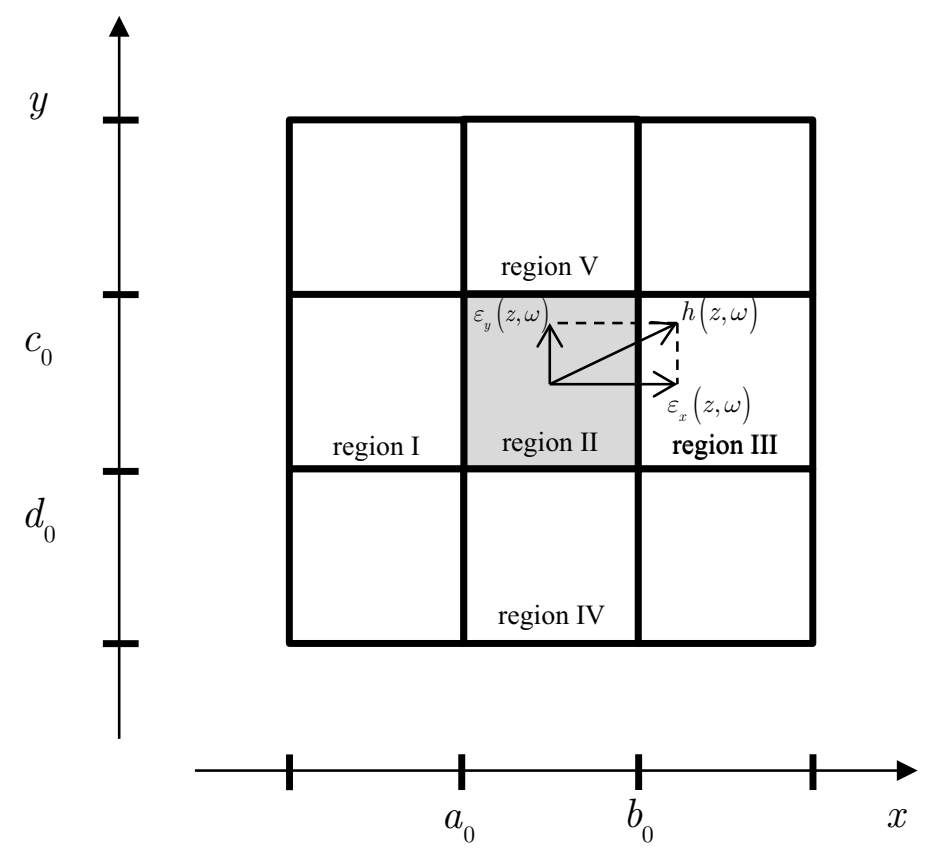

Fig. 1 Notations used for describing the vibrations of a homogeneous region (labelled II) in relation to four neighboring homogeneous regions (labelled I, III, IV and V).

\subsection{Control rod vibrations}

The modelling of control rod vibrations can be relatively easily carried out using the model of Williams [6] in the so-called weak absorber formulation of Pázsit [7]. The vibration of a control rod is assumed to be described by a one-dimensional structure along the $z$-direction vibrating perpendicularly to the twodimensional $(x, y)$ plane. Furthermore, the vibrating rod is assumed to always remain parallel to itself and to have the most significant effect on the thermal macroscopic absorption cross-section. In those conditions, the vibration of the rod will create a perturbation of the thermal absorption cross-section that is represented as:

$$
\delta \Sigma_{a, 2}(\mathbf{r}, t)=\gamma \Theta\left(z-z_{0}\right)\left[\delta\left(\mathbf{r}_{x y}-\mathbf{r}_{p, x y}-\varepsilon(t)\right)-\delta\left(\mathbf{r}_{x y}-\mathbf{r}_{p, x y}\right)\right]
$$

where $\gamma$ is the strength of the perturbation, $\mathbf{r}_{p, x y}$ is the equilibrium position of the rod in the $(x, y)$ plane, $\varepsilon(t)$ is a vector representing the displacement of the rod from its equilibrium position in the $(x, y)$ plane, and $z_{0}$ represents the axial elevation at which the rod is inserted (insertion from the top of the core). $\Theta\left(z-z_{0}\right)$ is the Heaviside function. Using a one-term Taylor expansion for Eq. (8) and after some algebra, the corresponding induced neutron noise is given as:

$$
\delta \phi_{g}(\mathbf{r}, \omega)=-\gamma \varepsilon(\omega) \cdot \nabla_{\mathbf{r}_{p, x y}} \hat{G}_{2 \rightarrow g}\left(\mathbf{r}, \mathbf{r}_{p, x y}, \omega\right)
$$

with

$$
\hat{G}_{2 \rightarrow g}\left(\mathbf{r}, \mathbf{r}_{x y}{ }^{\prime}, \omega\right)=\int G_{2 \rightarrow g}\left(\mathbf{r}, \mathbf{r}_{x y}{ }^{\prime}, z^{\prime}, \omega\right) \Theta\left(z^{\prime}-z_{0}\right) \phi_{2,0}\left(\mathbf{r}_{x y}{ }^{\prime}, z^{\prime}\right) d z^{\prime}
$$




\subsection{Core barrel vibrations}

Several modes of vibrations are possible for the core barrel. In the following, only the so-called beam or pendular mode is considered. Because the typical frequency of this mode overlaps with the frequencies of other phenomena, its detection remains challenging, explaining the focus on this mode. Two types of beam or pendular motions can occur: the so-called in-phase and out-of-phase modes [5]. These modes result from the interplay between the oscillations of the reactor pressure vessel with respect to the environment and the oscillations of the core barrel with respect to the reactor pressure vessel, to which the core barrel is attached. In the in-phase mode, the two oscillators move in the same directions, whereas in the out-of-phase mode, the two oscillators move in opposite directions.

For both modes of pendular or beam motions of the core barrel, the axial shape of the displacement of the active core with respect to the environment can be described in the same manner as a linear variation from the bearing points of the reactor pressure vessel downwards. From a neutronic viewpoint, the modelling of the pendular mode can be performed much alike the modelling of fuel assembly vibrations described in Section 2.3. Core barrel vibrations can be considered as the relative motion of the active fuel core region with respect to the reflector. Core barrel vibrations can thus be seen as a collective movement of all fuel assemblies with respect to the reflector. The perturbations introduced by core barrel vibrations can be considered as localized perturbations taking place at the boundary between the active core region and the reflector region.

\section{ANOMALY CLASSIFICATION AND LOCALIZATION}

After modelling the neutron noise induced by the above noise sources, the neutron noise was used for training, validating, and testing non-parametric unfolding techniques. Since, in some cases, a deterministic model of the noise source cannot be formulated, the Auto- and Cross-Power Spectral Densities (APSD/CPSD) were used to define the noise sources and the corresponding induced neutron noise. More information about the corresponding procedures and the definition of the statistical properties of the noise sources can be found in [8]. In order to investigate systems as representative as possible of commercial reactors, the thermal induced neutron noise was assumed to be measurable in only eight strings of detectors, each containing six axial neutron detectors, i.e. in total 48 detectors. Although CORE SIM + simulations provide both thermal and fast neutron noise, only the thermal noise is considered hereafter because neutron detectors are mostly sensitive to the thermal neutron flux. The dataset $\mathcal{D}=\left\{\left(X_{i}, \mathbf{y}_{i}^{c}, \mathbf{y}_{i}^{p}\right) ; i=1, \ldots, N\right\}$ was comprised of $N$ data examples $X_{i}$ corresponding to the aforementioned noise sources, each with an associating source coordinate and noise type label, $\mathbf{y}_{i}^{c}$ and $\mathbf{y}_{i}^{p}$ respectively. Each noise response signal $X_{i}$ is a $34 \times 32 \times 32$ complex volume which was further decomposed into its amplitude and phase components then concatenated channel-wise resulting in a $2 \times 34 \times 32 \times 32$ for compatibility with deep learning approaches. All but the 48 detector $i, j, k$ positions were set to zero to mimic the modelled reactor core. Each perturbation source label $\mathbf{y}_{i}^{c}$ is a vector of coordinates $(i, j, k)$ relative to $X_{i}$, and each type label $\mathbf{y}_{i}^{p}$ is a one-hot encoded vector of the perturbation. For the vibration-related perturbations, each possible vibration mode is considered as a different type. The task is to regress where perturbations originate within each $X_{i} 34 \times 32 \times 32$ volume and classify its type. In order to achieve this, we employed machine learning techniques, more specifically deep learning. Machine learning is a set of powerful algorithmic approaches for the processing of data, learning representations to detect or classify patterns, in an iterative manner without being explicitly programmed. Deep learning - a subset of machine learning - transforms the input to more complex and highly abstract representations through the nesting of hierarchical concepts, by stacking multiple Artificial Neural Network (ANN) layers [9]. 


\subsection{3-D Convolutional Neural Network}

Given the volumetric nature of the signal response to noise sources, it is necessary to capture spatial patterns within the input to classify type characteristics and localize the source coordinates. To achieve this, 3-D CNNs have been utilized, performing automatic feature extraction through a series of volume-wise convolutions and feature routing. Formally, an activated value of a given unit $\mathbf{A}_{i j k}^{\ell}$ at $(i, j, k)$ position in a 3D feature map of layer $\ell$ is calculated by summing the weighted kernel contributions from the previous layer units in $\mathbf{A}^{\ell-1}$ as:

$$
\mathbf{A}_{i j k}^{\ell}=\phi\left(\sum_{x=1}^{X} \sum_{y=1}^{Y} \sum_{z=1}^{Z} \mathbf{W}_{x y z}^{\ell} \odot \mathbf{A}_{i+x, j+y, k+z}^{\ell-1}\right)
$$

where $\mathbf{W}_{x y z}^{\ell}$ is a single learnt weight belonging to kernel $\mathbf{W}^{\ell}$ of dimensions $X \times Y \times Z$ in layer $\ell$, which is convolved with activations of the previous layer $\mathbf{W}^{\ell} \odot \mathbf{A}^{\ell-1}$, and $\phi(\cdot):=\max (0, \cdot)$ is a non-linear $\operatorname{ReLU}$ activation function. The bias terms have been omitted for simplicity. Fig. 2 depicts the volumetric convolution operation for a single convolution on the input signal (see further details in [2]). The network shown in the figure and proposed in this project is a modified DenseNet network [10], replacing 2-D convolutions and pooling layer for strided 3-D convolutions to accommodate the volumetric input and retaining spatial information down-scaling through strides. The network is comprised of three dense blocks, which allow for a greater flow of information through the network via the concatenation of all previous feature maps within the block to the current feature maps. This results in a reduction of vanishing gradients during the backpropagation procedure improving stability and performance. The network was trained with backpropagation updating the weights via gradient descent minimizing a multi-objective (classification and regression) criterion of a linear weighted sum of losses:

$$
\mathcal{L}\left(\mathcal{D} ; \mathbf{W}, \lambda_{1}, \lambda_{2}\right)=-\frac{1}{M} \sum_{i=1}^{M}\left[\frac{\lambda_{1}}{P} \sum_{j=1}^{P}\left[\mathbf{y}_{j} \log \left(\hat{\mathbf{y}}_{j}\right)+\left(1-\mathbf{y}_{j}\right) \log \left(1-\hat{\mathbf{y}}_{j}\right)\right]-\frac{\lambda_{2}}{C} \sum_{c=1}^{C}\left\|\mathbf{y}_{c}-\hat{\mathbf{y}}_{c}\right\|^{2}\right],
$$

where $M$ is the size of mini-batch given the sum of losses per task, $P$ and $C$ denote the number of perturbation types and location coordinates $(i, j, k)$ respectively, with $\lambda_{1}$ and $\lambda_{2}$ being manually tuned weight coefficients controlling the priority of each task to the update of the network weights.

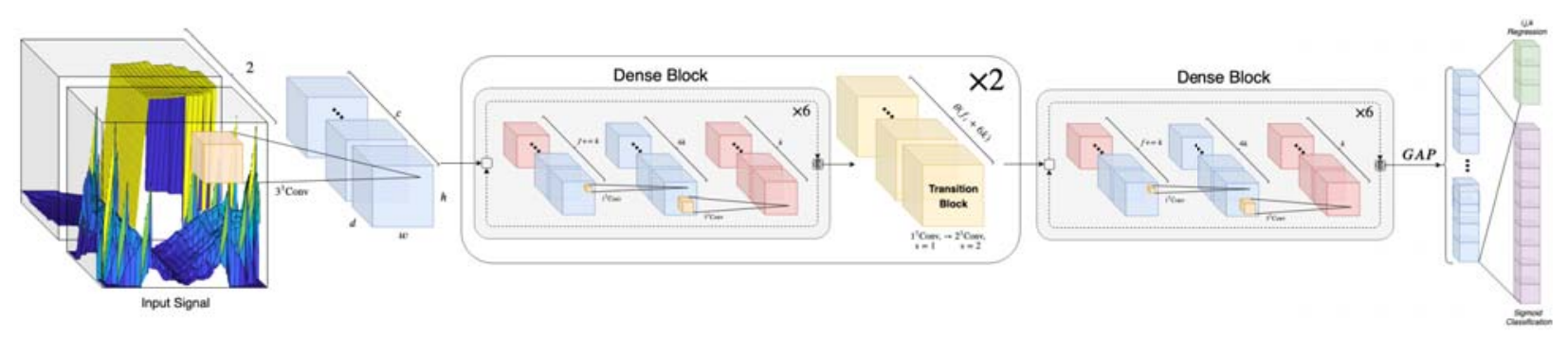

Fig. 2 The proposed 3D DenseNet architecture, comprised of 3 dense blocks each containing 6 layers per block and growth rate of $k=32$. Each unit in the output layer represents a classification perturbation type or source location $(i, j, k)$. 


\subsection{Experimental Results}

The results of the experiments conducted can be seen in Table I. The first experiment investigated the effect of a reduction in training dataset size, simulating the real case of limited training data (second, third and fourth rows of the given results). The dataset is divided into train, validation and test sets, $\mathcal{D}_{\text {Train }}, \mathcal{D}_{\text {Valid }}, \mathcal{D}_{\text {Test }}$ respectively, where the sets contain no overlapping source $i, j, k$ perturbation labels between them. The result of which shows a very minimal impact in performance of classification (see the F1-score values in the Table) and a small increase in localization mean squared error (MSE) and mean absolute error (MAE). Furthermore, the robustness of the proposed system has been experimented through the addition of white Gaussian noise at two different signal-to-noise-ratios (SNR), demonstrated in the second, fifth and sixth rows of Table I. The effect of noise addition is less impactful than the reduction in training data showing the robustness of the system to additive noise.

Table I. Comparative results of the proposed 3D DenseNet: $(\cdot)$ denotes the SNR of the noise added. Each model was run three times and the results are given as an average and standard deviation.

\begin{tabular}{c|c|c|c|c|c}
\hline \multirow{2}{*}{ Sensors } & \multirow{2}{*}{$\begin{array}{c}\text { Train / Valid } \\
\text { / Test }(\%)\end{array}$} & \multicolumn{2}{|c|}{ Classification } & \multicolumn{2}{c}{ Regression $(i, j, k)$} \\
\cline { 3 - 6 } & $70 / 15 / 15$ & $0.06 \pm 0.051$ & $0.9344 \pm 0.004$ & $0.1435 \pm 0.011$ & $0.0342 \pm 0.008$ \\
\hline All & $70 / 15 / 15$ & $\mathbf{0 . 1 1} \pm \mathbf{0 . 0 1 0}$ & $\mathbf{0 . 9 3 1 1} \pm \mathbf{0 . 0 0 1}$ & $\mathbf{0 . 2 9 0 2} \pm \mathbf{0 . 0 1 1}$ & $\mathbf{0 . 3 0 7 2} \pm \mathbf{0 . 0 1 4}$ \\
48 detectors & 70 (\%) & F1-Score & MAE & MSE \\
48 detectors & $25 / 15 / 60$ & $0.32 \pm 0.025$ & $0.9149 \pm 0.002$ & $0.3978 \pm 0.017$ & $0.6407 \pm 0.052$ \\
48 detectors & $15 / 25 / 60$ & $0.44 \pm 0.061$ & $0.9141 \pm 0.003$ & $0.4858 \pm 0.017$ & $0.7727 \pm 0.006$ \\
48 detectors (3) & $70 / 15 / 15$ & $0.15 \pm 0.006$ & $0.9231 \pm 0.001$ & $0.3456 \pm 0.016$ & $0.4905 \pm 0.011$ \\
48 detectors (1) & $70 / 15 / 15$ & $0.19 \pm 0.036$ & $0.9225 \pm 0.002$ & $0.3709 \pm 0.020$ & $0.5185 \pm 0.017$ \\
\hline
\end{tabular}

\section{CONCLUSIONS}

The proposed approach demonstrates the potential of machine learning methodologies for core monitoring and diagnostic purposes, and more precisely for the classification and, when relevant, for the localization of anomalies. The developed methodology relies on the pre-calculation of the neutron flux responses in the frequency domain to specified stationary perturbations. Machine learning is thereafter used for unfolding from the local neutron flux responses at very few core locations the driving perturbation. Low error rates are achieved in all cases even under the conditions of the addition of noise and few training examples to learn from. The development of extensions to this approach are to be made to handle varying numbers of simultaneous perturbations, and further validation given real reactor readings is essential to show the capability of the system in practice.

\section{ACKNOWLEDGMENTS}

The research conducted was made possible through funding from the Euratom research and training programme 2014-2018 under grant agreement No 754316 (CORTEX project). 


\section{REFERENCES}

1. F. Calivà, F. De Sousa Ribeiro, A. Mylonakis, C. Demazière, P. Vinai, G. Leontidis and S. Kollias, "A deep learning approach to anomaly detection in nuclear reactors," Proceedings of 2018 International Joint Conference on Neural Networks (IJCNN2018), Rio de Janeiro, Brazil, July 8-13, 2018 (2018).

2. F. De Sousa Ribeiro, F. Calivà, D. Chionis, A. Dokhane, A. Mylonakis, C. Demazière, G. Leontidis and S. Kollias, "Towards a deep unified framework for nuclear reactor perturbation analysis," Proceedings of the IEEE Symposium Series on Computational Intelligence (SSCI 2018), Bengaluru, India, November 18-21, 2018 (2018).

3. C. Demazière, P. Vinai, M. Hursin, S. Kollias and J. Herb, "Overview of the CORTEX project," Proceedings of the International Conference on the Physics of Reactors - Reactor Physics paving the way towards more efficient systems (PHYSOR2018), Cancun, Mexico, April 22-26, 2018 (2018).

4. A.G. Mylonakis, P. Vinai and C. Demazière C., "Neutron noise modelling for nuclear reactor core diagnostics," Proceedings of the 27th Symposium of the Hellenic Nuclear Physics Society, Athens, Greece, June 8-9, 2018 (2018).

5. C. Bläsius, private communication, Gesellschaft für Anlagen- und Reaktorsicherheit (GRS) gGmbH (2018).

6. M.M.R. Williams, Random processes in nuclear reactors, Pergamon Press, Oxford, United Kingdom (1974).

7. I. Pázsit, "Investigation of the space-dependent noise induced by a vibrating absorber," Atomkernenergie, 30, pp. 29-35 (1977).

8. C. Demazière and A. Dokhane, "Description of scenarios for the simulated data," Deliverable D3.1 of the CORTEX project (2019).

9. Y. LeCun, Y. Bengio and G. Hinton, “Deep learning," Nature, 521, pp. 436-444 (2015).

10. G. Huang, Z. Liu, L. Van Der Maaten and K. Q. Weinberger, "Densely connected convolutional networks." In Proceedings of the IEEE conference on computer vision and pattern recognition (CVPR 2017), Honolulu, HI, USA, July 21-26, 2017 (2017). 